\title{
Condition Monitoring of Lithium Polymer Batteries Based on a Sigma-Point Kalman Filter
}

\author{
Bo-Hwan Seo*, Thanh Hai Nguyen ${ }^{* *}$, Dong-Choon Lee ${ }^{\dagger}$, Kyo-Beum Lee $^{* * *}$, and Jang-Mok Kim ${ }^{* * * *}$ \\ *LG Electronics, Inc., Changwon, Korea \\ ${ }^{\dagger}{ }^{* *}$ Dept. of Electrical Engineering, Yeungnam University, Gyeongsan, Korea \\ ${ }^{* * *}$ Dept. of Electrical and Computer Engineering, Ajou University, Suwon, Korea \\ ${ }^{* *}$ School of Electrical Engineering, Pusan National University, Busan, Korea
}

\begin{abstract}
In this paper, a novel scheme for the condition monitoring of lithium polymer batteries is proposed, based on the sigma-point Kalman filter (SPKF) theory. For this, a runtime-based battery model is derived, from which the state-of-charge (SOC) and the capacity of the battery are accurately predicted. By considering the variation of the serial ohmic resistance $\left(R_{o}\right)$ in this model, the estimation performance is improved. Furthermore, with the SPKF, the effects of the sensing noise and disturbance can be compensated and the estimation error due to linearization of the nonlinear battery model is decreased. The effectiveness of the proposed method is verified by Matlab/Simulink simulation and experimental results. The results have shown that in the range of a SOC that is higher than $40 \%$, the estimation error is about $1.2 \%$ in the simulation and $1.5 \%$ in the experiment. In addition, the convergence time in the SPKF algorithm can be as fast as $300 \mathrm{~s}$.
\end{abstract}

Key Words: Capacity, Lithium polymer battery, Monitoring, Sigma-point Kalman filter, State-of-charge

\section{INTRODUCTION}

Recently, the development and utilization of renewable energy has received a great deal of attention due to environmental problems and a lack of fossil resources. As renewable energy is increasingly used, the interest in battery technologies is also increasing. Batteries are used in the energy storage systems for photovoltaic power generation, wind power generation, etc., and as an energy source for electric vehicle drives and uninterruptible power supplies (UPS) [1]-[4].

For For extending battery lifetime and preventing permanent damage, a battery management system (BMS), which monitors the parameters and maintains the battery performance at an optimal level, is usually needed. The two important parameters are the state-of-charge (SOC) and the state-of-health ( $\mathrm{SOH})$, where the SOC is defined as the ratio of the charged energy in a battery to the total energy that can be

Manuscript received Mar. 25, 2012; revised Jun. 22, 2012

Recommended for publication by Associate Editor Woo-Jin Choi.

†Corresponding Author: dclee@yu.ac.kr

Tel: +82-53-810-2582, Fax: +82-53-810-4767, Yeungnam University

*LG Electronics, Inc., Changwon, Korea

** Dept. of Electrical Eng., Yeungnam University, Korea

*** Dept. of Electrical and Computer Eng., Ajou University, Korea

**** School of Electrical Eng., Pusan National University, Korea stored in the battery, while the $\mathrm{SOH}$ represents the ability of a battery to store energy.

Direct measurement of the battery SOC is very difficult since the SOC is related to chemical states inside the battery. Therefore, the appropriate estimation method is usually used. For estimating the SOC, several methods have been proposed in [5]-[7]. In the current integration method (or ampere counting method), the charging or discharging currents are integrated [5]. This method is simple, but the estimation error is high due to a large accumulation of measurement errors. In addition, selecting an initial value accurately is hard. The opencircuit voltage (OCV) method gives very good performance [6], [7]. However, the open-circuit voltage test should be performed during the steady state of the battery. Therefore, online measurement is impossible. In the impedance measurement method [8], instruments for measuring the impedance of a battery are required, in which the on-line measurements are also difficult. The state estimation method using a Kalman filter includes the current integration method and the OCV method [9]. In this method, the sensing noise and disturbance are considered, so that the recursive algorithm can estimate the battery parameters more accurately. The drawback of this method is that an exact model is needed for accurate estimation. 
Although a lot of research results have been presented for estimating the SOC, studies for estimating the $\mathrm{SOH}$ or the available capacity of a battery are not common. The $\mathrm{SOH}$ is related to the replacement of a battery, in which the $\mathrm{SOH}$ indicates its aging and deterioration conditions. Studies on $\mathrm{SOH}$ can be classified into two groups. One group is based on the measurement of the battery capacity through the discharging test and using the impedance or conductance of the battery in comparison with the reference values [10]-[12]. The other group is based on the state estimation of a battery using an estimation algorithm [13]-[15].

In this paper, the SOC and capacity of a lithium polymer battery are estimated, based on a sigma-point Kalman filter. First, the parameters of the battery are obtained by tests. Then, a battery model with nonlinear characteristics is derived. For this model, the SPKF is applied to estimate the state variables of the SOC and the capacity. The advantage of this algorithm is that the SPKF compensates for the error of the battery model and the effect of noise, and reduces the linearization error in comparison with the EKF. The effectiveness of the proposed method is verified by simulation and experimental results, which show that the convergence time of $300 \mathrm{~s}$ in the SPKF algorithm is faster than that of $500 \mathrm{~s}$ in the EKF method.

\section{Model AND STATE EQUATIONS OF LiTHIUM BATTERIES}

\section{A. Lithium Battery Models}

For analyzing battery performance, different types of battery models have been developed. First, the electrochemical model is appropriate for optimizing the physical aspects and investigating the internal dynamics of batteries [16]. On the other hand, the mathematical model is not appropriate for representing physical characteristics [17]. The circuit-based model has often been used [18]-[21] since the battery characteristics are represented adequately and it is easy to use for analysis and simulation. For the battery model with nonlinear characteristics, the calculation time for the estimation algorithm increases and the real-time implementation is complex.

In this paper, the runtime-based battery model is adopted, which is shown in Fig. 1 [22]. This model is composed of two parts. Firstly, the battery lifetime part consists of a capacitance $\left(C_{\text {capacity }}\right)$ and a current source depending on the discharging/charging current, which models the capacity of the battery. Secondly, the Thevenin-equivalent circuit is composed of $V_{\text {oc(Vsoc) }}$ which represents the relationship between the SOC and the OCV (open-circuit voltage), $R_{o}$ which represents the equivalent resistance, and the $R_{1} C_{1}$ parallel circuit which represents the diffusion and polarization effects. The identification of each parameter is described in Section IV.

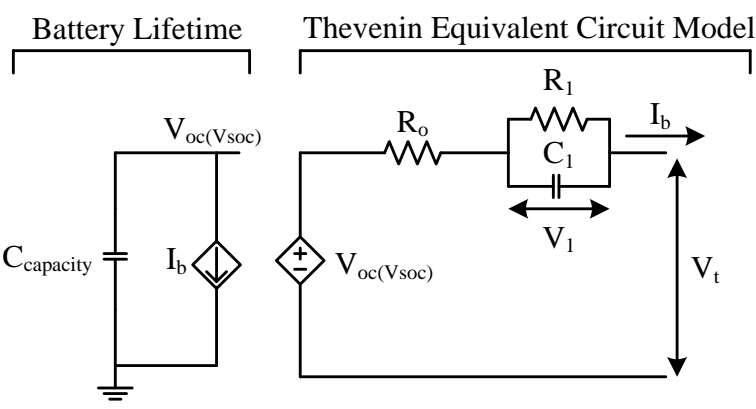

Fig. 1. Runtime-based battery model.

\section{B. State Equations of a Battery}

From Fig. 1, the terminal voltage of a battery is expressed as:

$$
V_{t}=V_{o c(V s o c)}-R_{o} I_{b}-V_{1}
$$

where $V_{t}$ is the terminal voltage of the battery, $V_{1}$ is the voltage across the $R_{1} C_{1}$ parallel circuit, $I_{b}$ is the discharging/charging current, and $V_{\text {oc(Voc) }}$ is the open-circuit voltage.

From the battery lifetime part, the derivative of the opencircuit voltage is derived as:

$$
\dot{V}_{\text {oc(Vsoc) }}=-\frac{I_{b}}{C_{\text {capacity }}} .
$$

The discharging current $I_{b}$ consists of $I_{R 1}$ and $I_{C 1}$, which flow through $R_{1}$ and $C_{1}$, respectively. Hence:

$$
I_{b}=I_{R 1}+I_{C 1} .
$$

Then, the relationship between the voltage and current of the diffusion capacitor gives:

$$
\dot{V}_{1}=\frac{1}{C_{1}} I_{C 1}
$$

From (2)-(4), the derivative of the voltage across the diffusion capacitance is rewritten as:

$$
\dot{V}_{1}=-\frac{1}{R_{1} C_{1}} V_{1}+\frac{1}{C_{1}} I_{b}
$$

From (2) and (5), the state equation can be expressed as:

$$
\dot{x}=f(x, u)=\left[\begin{array}{c}
\dot{V}_{o c(V s o c)} \\
\dot{V}_{1}
\end{array}\right]=\left[\begin{array}{c}
-\frac{I_{b}}{C_{\text {capacity }}} \\
-\frac{1}{C_{1} R_{1}} V_{1}+\frac{1}{C_{1}} I_{b}
\end{array}\right]
$$

where $x=\left[\begin{array}{ll}V_{o c(V s o c)} & V_{1}\end{array}\right]^{T}$ is a state vector and $u=I_{b}$ is an input. The output y can be expressed as:

$$
y=h(x, u)=V_{t}=V_{o c(V s o c)}-R_{o} I_{b}-V_{1} .
$$




\section{PROPOSED APPROACH}

\section{A. Sigma-point Kalman Filter Algorithm}

The SPKF is one of the Kalman filter algorithms, which is suitable for nonlinear system applications [23]-[24]. In the SPKF, scalar scaling parameters are used for selecting several sigma points, which are a set of weighted samples capturing the true mean and covariance of prior random variables. The sigma-points are propagated through true nonlinear functional evaluations to generate a posterior sigma-point set. The transformed mean and covariance are calculated by the transformed sigma points. In this computation progress, the SPKF does not include a linearization process like the firstorder Taylor series expansion in the EKF method. In summary, the SPKF has some advantages when compared with the EKF:

1) The linearization error of the system is reduced since the linearization process is not needed.

2) In the EKF method, the calculation of a Jacobian matrix is required. Thus the model becomes more complex and its implementation is more difficult.

Therefore, in this paper, the SPKF is used for estimating the SOC and capacity of batteries.

In general, the state equations for estimation are expressed as [25]:

$$
\begin{aligned}
& x_{k}=f\left(x_{k-1}, u_{k-1}\right)+w_{k-1} \\
& y_{k}=h\left(x_{k}, u_{k-1}\right)+v_{k-1}
\end{aligned}
$$

where $f$ is a process model, $h$ is a measurement model, $x$ is the selected state vector, $u$ is the input vector, $y$ is the measurable output vector, $w$ is the process noise and $v$ is the measurement noise.

The SPKF algorithm is described as follows [23]-[24]:

1) Initialization: The initial values of the state $x_{0}$ and the covariance $P_{o}$ are selected as:

$$
\begin{aligned}
& \hat{x}_{0}=E\left[x_{0}\right] \\
& P_{o}=E\left[\left(x_{0}-\hat{x}_{0}\right)\left(x_{0}-x_{0}\right)^{T}\right]
\end{aligned}
$$

where " $\wedge$ " denotes the estimated value and $E$ indicates the expected value.

The augmented state vector $x^{a}$ and the covariance $P^{a}$ are used for increasing the accuracy of the SPKF.

$$
\begin{gathered}
x^{a}=\left[\begin{array}{lll}
x^{T} & w^{T} & v^{T}
\end{array}\right] \\
\hat{x}_{0}^{a}=E\left[x_{0}^{a}\right]=E\left[\begin{array}{lll}
x_{0} & 0 & 0
\end{array}\right]^{T} \\
P_{0}^{a}=E\left[\left(x_{0}^{a}-\hat{x}_{0}^{a}\right)\left(x_{0}^{a}-x_{0}^{a}\right)^{T}\right]=\left[\begin{array}{ccc}
P_{0} & 0 & 0 \\
0 & R_{v} & 0 \\
0 & 0 & R_{n}
\end{array}\right]
\end{gathered}
$$

where the dimension $n$ of the augmented state vector $x^{a}$ is the sum of the dimensions of the states, the process noise $w$ and the measurement noise $v$.

2) Calculating the sigma points and weights: The number of sigma points, $\chi^{a}$, is $2 n+1$ and each sigma point is calculated as follows:

$$
\begin{aligned}
& \chi_{0}^{a}=\hat{x}_{k-1}^{a} \\
& \chi_{i}^{a}=\hat{x}_{k-1}^{a}+\left(h \sqrt{P_{x, k-1}^{a}}\right)_{i}, i=1, \ldots, n \\
& \chi_{i}^{a}=\hat{x}_{k-1}^{a}-\left(h \sqrt{P_{x, k-1}^{a}}\right)_{i}, i=n+1, \ldots, 2 n
\end{aligned}
$$

where $h$ is a scaling parameter for the SPKF, which is positive. The weights $\omega$ for calculating the transformed mean and covariance are computed as:

$$
\begin{aligned}
& \omega_{0}=\frac{h^{2}-n}{h^{2}} \\
& \omega_{i}=\frac{1}{2 h^{2}}, i=1, \ldots, 2 n .
\end{aligned}
$$

3) Update of the state estimation: The estimated mean $\hat{x}_{k}^{-}$ and the covariance $P_{x_{k}}^{-}$are calculated by using the predicted values of $k^{\text {th }} \quad \chi_{k \mid k-1}^{a}$ through the process model $f$ as:

$$
\begin{aligned}
& \chi_{k \mid k-1}^{a}=f\left(\chi_{k-1}^{x}, \chi_{k-1}^{v}, u_{k-1}\right) \\
& \hat{x}_{k}^{-}=\sum_{i=0}^{2 n} \omega_{i} \times \chi_{i, k \mid k-1}^{a} \\
& P_{x_{k}}^{-}=\sum_{i=0}^{2 n} \omega_{i} \times\left(\chi_{i, k \mid k-1}^{x}-\hat{x}_{k}^{a}\right)\left(\chi_{i, k \mid k-1}^{x}-x_{k}^{-}\right)^{T} .
\end{aligned}
$$

4) Measurement update: From the estimated values of $k^{\text {th }}$ $\chi_{k \mid k-1}^{a}$ and the measurement model $h$, the predicted measurement values $\hat{y}_{k}^{-}$and the corresponding covariance are calculated as:

$$
\begin{aligned}
& Y_{k \mid k-1}=h\left(\chi_{k \mid k-1}^{x}, \chi_{k-1}^{n}\right) \\
& \hat{y}_{k}^{-}=\sum_{i=0}^{2 n} \omega_{i} \times Y_{i, k \mid k-1} \\
& P_{\tilde{y}_{k}}=\sum_{i=0}^{2 n} \omega_{i} \times\left(Y_{i, k \mid k-1}-\hat{y}_{k}^{\hat{n}}\right)\left(Y_{i, k \mid k-1}-y_{k}^{-}\right)^{T} \\
& P_{x_{k} y_{k}}=\sum_{i=0}^{2 n} \omega_{i} \times\left(\chi_{i, k \mid k-1}^{x}-\hat{x}_{k}\right)\left(Y_{i, k \mid k-1}-y_{k}^{-}\right)^{T} .
\end{aligned}
$$


For correcting the estimated mean and the covariance, the Kalman gain $K_{k}$ is used, which is expressed as:

$$
K_{k}=P_{x_{k} y_{k}} P_{y_{k}}^{-1}
$$

Finally, the measurement update and the corresponding covariance matrix are calculated as:

$$
\begin{gathered}
x_{k}=x_{k}^{-}+K_{k}\left(y_{k}-\hat{y}_{k}^{-}\right) \\
P_{x_{k}}=P_{x_{k}}^{-}-K_{k} P_{\tilde{y}_{k}} K_{k}^{T} .
\end{gathered}
$$

\section{B. Estimation of the SOC and Capacity Using SPKF}

The available capacity of the battery is modeled as a full-

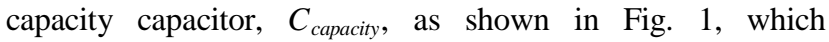
represents the entire charge stored in the battery. The capacity of the battery is denoted in terms of Farad (F), which is expressed as:

$$
C_{\text {capacity }}=3600 \cdot \text { Capacity }_{\text {initial }} \cdot f_{1}(\text { cycle }) \cdot f_{2}(\text { temp. })
$$

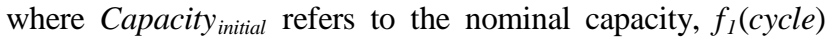
represents a reduction ratio of the capacity due to the charging and discharging cycles, and $f_{2}$ (temp.) denotes a capacity reduction ratio according to temperature variations. In this paper, $f_{2}$ (temp.) is assumed to be unity since the experiment is performed at about $25{ }^{\circ} \mathrm{C}$. Therefore, the available battery capacity can be rewritten as:

$$
C_{\text {capacity }}=3600 \cdot \text { Capacity }_{\text {initial }} \cdot f_{1}(\text { cycle }) .
$$

Then, from (31), the available battery capacity can be estimated. The augmented state equation is arranged as follows:

$$
\begin{gathered}
\dot{x}=f(x, u)=\left[\begin{array}{c}
\dot{V}_{o c(V s o c)} \\
\dot{V}_{1} \\
\left(\frac{1}{C_{\text {capacity }}}\right)
\end{array}\right]=\left[\begin{array}{c}
-\frac{I_{b}}{C_{\text {capacity }}} \\
-\frac{1}{C_{1} R_{1}} V_{1}+\frac{1}{C_{1}} I_{b} \\
0
\end{array}\right] \\
y=h(x, u)=V_{t}=V_{o c(V s o c)}-R_{o} I_{b}-V_{1}
\end{gathered}
$$

where the state vector is $x=\left[\begin{array}{lll}V_{o c(V s o c)} & V_{1} & \frac{1}{C_{\text {capacity }}}\end{array}\right]^{T}$, the input is $u=I_{b}$, and the output is $y=V_{t}$.

By estimating the state variable $V_{o c(V s o c)}$, the SOC can be obtained from the relation between the open-circuit voltage and the SOC, which is described in the following section.

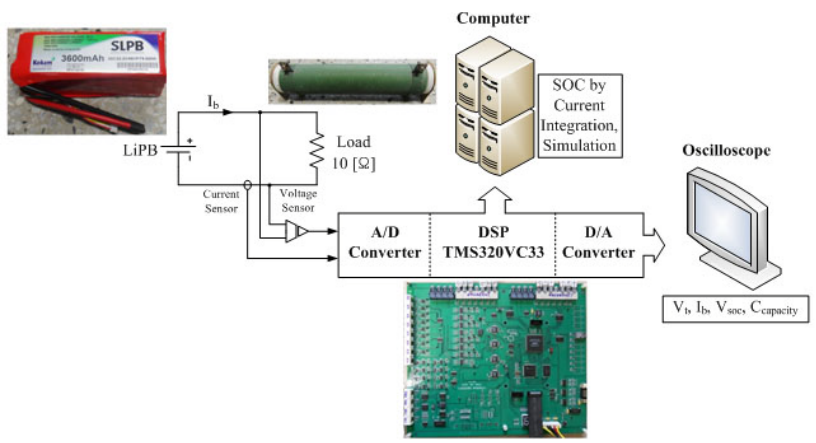

Fig. 2. Experimental setup.

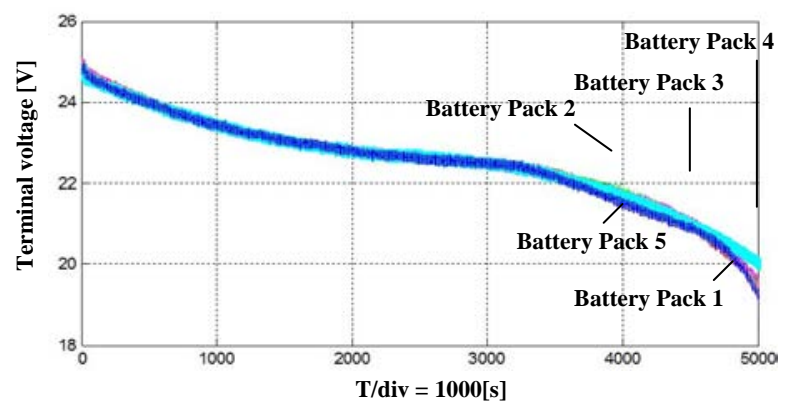

Fig. 3. Terminal voltages of five battery packs during discharging.

\section{PARAMETER IDENTIFICATION OF LITHIUM BATTERIES}

\section{A. Setup for Test}

In order to identify the battery parameters, a battery test system has been set up as shown in Fig. 2, where a Kokam HEP06S01Pl lithium polymer battery was adopted. The nominal capacity of the battery is $3.6 \mathrm{Ah}$ and its nominal voltage is $22.2 \mathrm{~V}$.

The discharging test is performed up to a nominal enddischarged voltage of $18 \mathrm{~V}$. The measured discharging voltage and current are used for the estimation. The discharging test is done through a resistive load for ease of analysis, which is selected as $10 \Omega$.

\section{B. Battery Terminal Voltages}

During the discharging tests, the curves of the battery terminal voltage have been investigated for five battery packs, as shown in Fig. 3. It can also be seen that each battery terminal voltage for discharging is slightly different and greatly decreased after 3,200 s. In this paper, the fifth battery pack is chosen for investigations.

C. $V_{o c(V s o c)}$

The OCV reflects the electrochemical characteristics of the active electrode materials in the battery, which depend on the SOC. In order to indentify the open-circuit voltage, first, the terminal voltage is measured for the battery in the fully charged state, which is the terminal voltage at $100 \%$ of the 


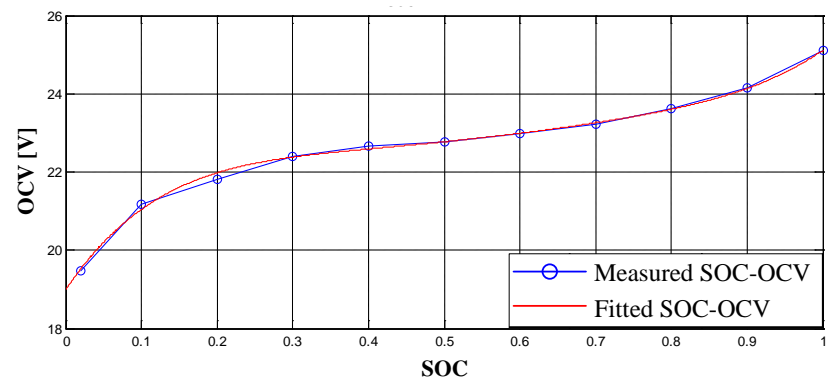

Fig. 4. Open-circuit voltage versus SOC (measured data).

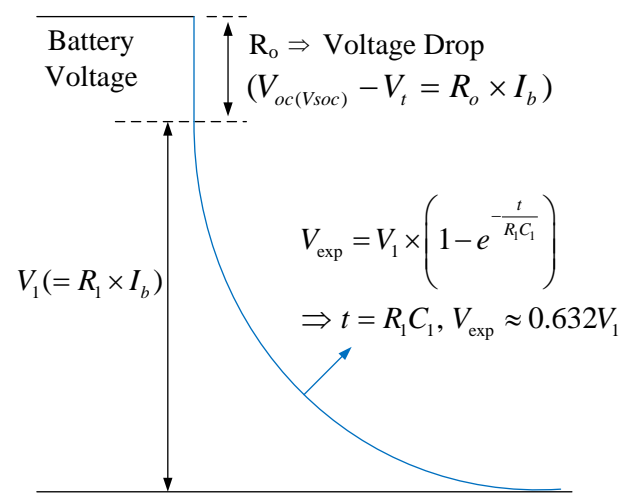

Fig. 5. Parameter identification.

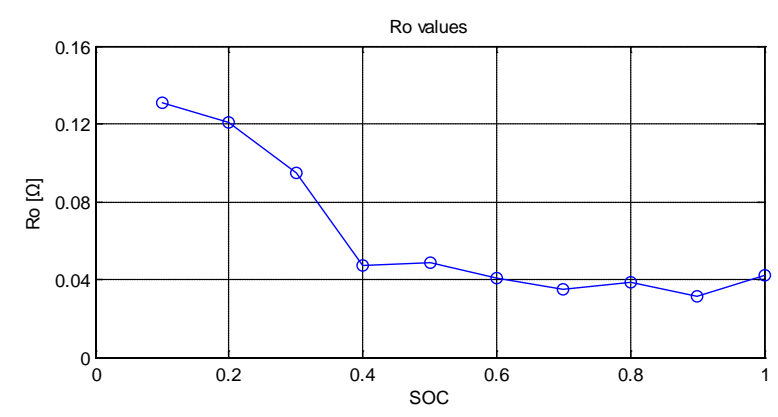

Fig. 6. Relationship of $\mathrm{R}_{\mathrm{o}}$ and SOC.

SOC. Next, the battery is discharged by up to $90 \%$ of the nominal capacity. Then, it remains in the open-circuit condition. The terminal voltage can be considered as the open-circuit voltage after 3,600 s when the battery reaches the steady state, where the change of the terminal voltage is negligible. Repetitively, the open-circuit voltages at other SOC values of the battery are obtained. Fig. 4 shows the relationship between the OCV and the SOC. A nonlinear equation of the OCV resulting from curve fitting, which is a function of the SOC, is used for the SPKF algorithm.

\section{D. $R_{o}, R_{1}, C_{1}$}

The parameters for the battery model can be calculated with a help of Fig. 5, in which the voltage behavior is illustrated during discharging. At the beginning of the
TABLE I

PARAMETERS OF BATTERY MODEL

\begin{tabular}{|c|c|}
\hline Parameters & Values \\
\hline$R_{o}$ & $41.9[\mathrm{~m} \Omega]$ \\
\hline$R_{1}$ & $22.3[\mathrm{~m} \Omega]$ \\
\hline$C_{1}$ & $228.7[\mathrm{~F}]$ \\
\hline
\end{tabular}

(a)

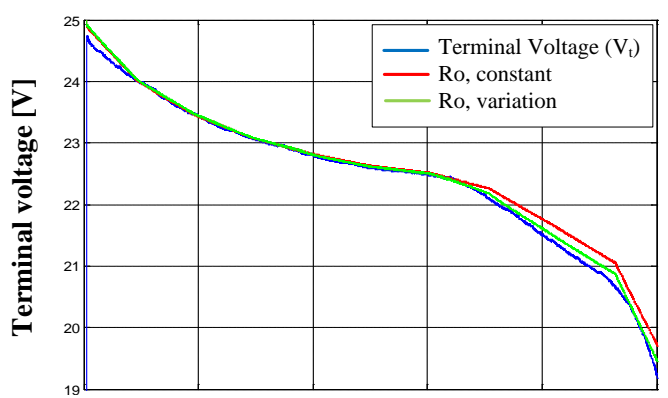

(b)

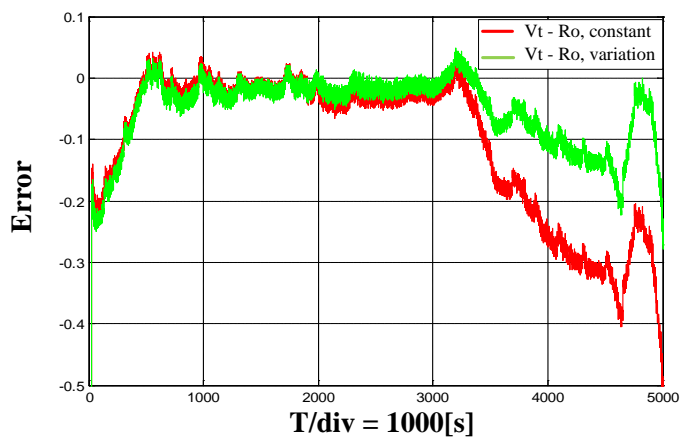

Fig. 7. Comparison of estimation performance with a constant and variable of $R_{0}$. (a) Terminal voltage. (b) Errors.

discharging process, the difference between the OCV and the terminal voltage is applied across the equivalent resistance $R_{0}$ since the initial voltage of the diffusion capacitor is zero. The diffusion resistance $R_{1}$ is calculated from the discharging current and its voltage $V_{1}$. Meanwhile, the diffusion capacitance $C_{1}$ is computed from the exponential voltage drop when the capacitor reaches the steady state. The identified parameters for the Kokam HEP06S01P lithium battery are shown in Table I.

Fig. 6 shows that the equivalent resistance $R_{o}$ depends on the SOC values. This resistance varies a little over the range of $40 \%$ of the SOC to the full change condition, and then increases rapidly during further discharging. Due to the variation of $R_{0}$ with regard to the SOC values, the estimation error is increased if $R_{0}$ is treated as a constant for the estimation algorithm.

Fig. 7 shows a comparison of the two cases of the constant and variable of $R_{0}$. Fig. 7(a) shows the actual terminal voltage and its values for the model in the two different cases. Fig. 7(b) shows the errors between them, in which the error in the case of the variable $R_{0}$ is lower than that in the case of the constant $R_{0}$. 
(a)

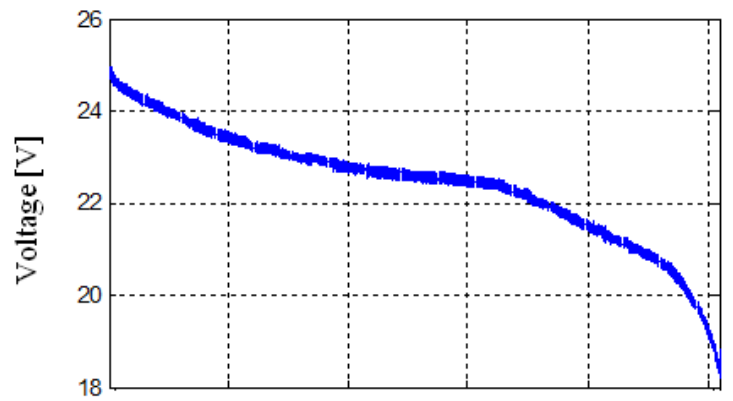

(b)

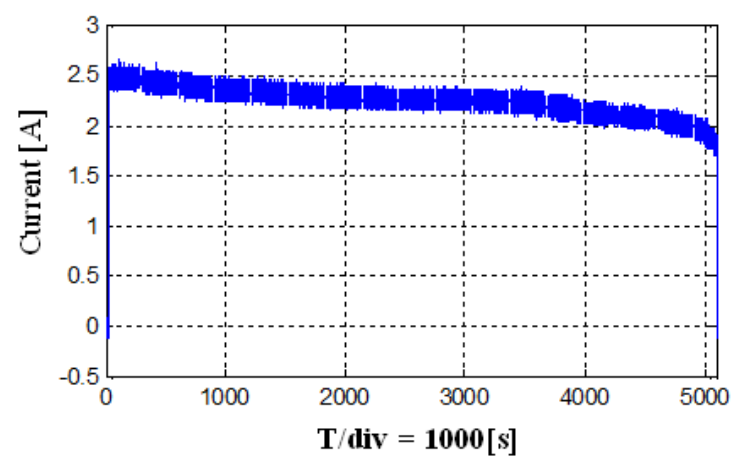

Fig. 8. Discharging test. (a) Terminal voltage. (b) Current.

(a)

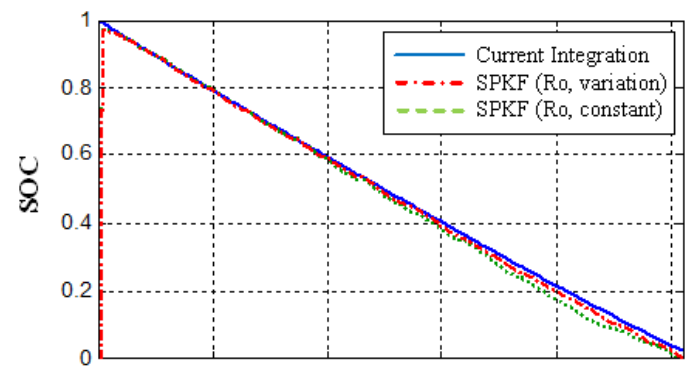

(b)

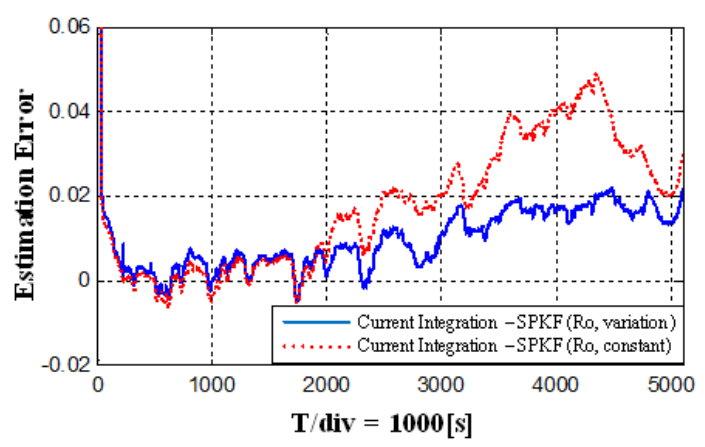

Fig. 9. Performance of estimated SOC. (a) SOC values. (b) Errors.

\section{Simulation RESUlts}

To verify the effectiveness of the proposed method, simulations using Matlab/Simulink have been carried out, in which the test system, as shown in Fig. 2, is modeled. The battery parameters are also the same, as identified in section IV. The sampling time for the measured voltage and current is chosen as $1 \mathrm{~s}$. (a)

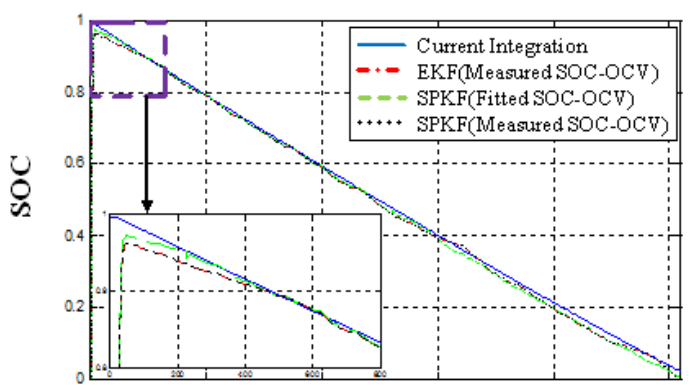

(b)

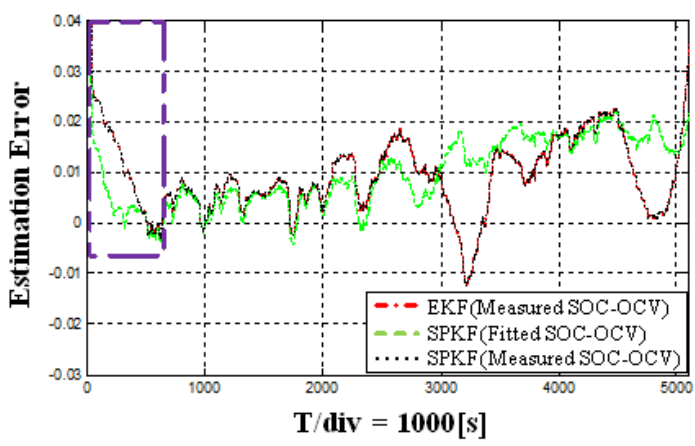

Fig. 10. Comparison of SOC estimation methods. (a) Estimated SOCs. (b) Estimation errors.

Fig. 8 shows the discharging test with a resistive load of 10 $\Omega$. For this, the terminal voltage is decreased as shown in Fig. 8(a). In addition, the discharging current is decreased due to a constant load, as shown in Fig. 8(b).

Fig. 9 shows the estimated performance of the SOC using the SPKF, in which the two cases of using the constant and the variable $R_{o}$ are considered. Fig. 9(a) shows the SOC values during the discharging process with the SPKF algorithm and with the current integration method. The estimated errors in the cases of using the SPKF algorithm with a constant $R_{0}$ or a variation $R_{0}$, are shown in Fig. 9(b), in which the SOC value resulting from the current integration method is a reference. The estimation error begins to increase after $2000 \mathrm{~s}$ and the maximum error of $2.5 \%$ in the case of variable $R_{0}$ is lower than the $5 \%$ maximum error in the case of a constant $R_{0}$.

The comparison of the estimation performance of the SOC with the EKF and SPKF algorithms is shown in Fig. 10. In this investigation, the variable $R_{o}$ values in the battery model are applied to the SPKF algorithm, and the measured relationship of the SOC versus the OCV is used for the EKF algorithm [9]. The estimation errors for the EKF and SPKF methods are acceptable at less than about 2.5\%, as shown in Fig. 10(b). However, the convergence time in the SPKF algorithm is about $300 \mathrm{~s}$ which is shorter than that of the EKF algorithm with about 500 s, as shown in Fig. 10(a). In addition, it can be seen from the estimation error in Fig. 10(b) that the performance in the case of using the SPKF algorithm is better than that in the case of using the EKF for $40 \sim 100 \%$ of the SOC. 


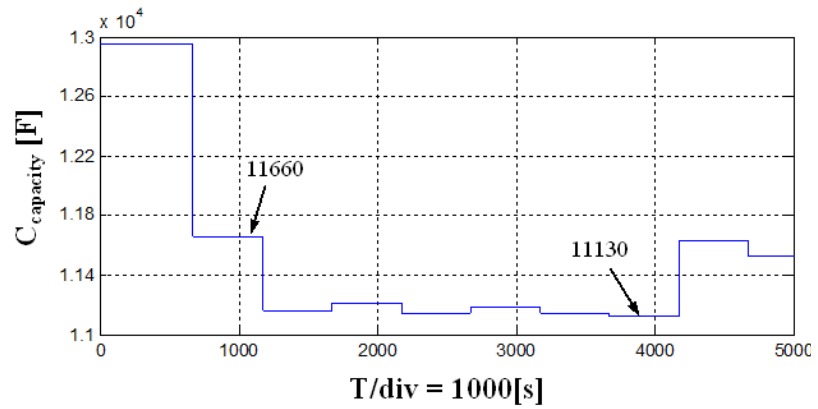

Fig. 11. Estimated $C_{\text {capacity }}$

(a)

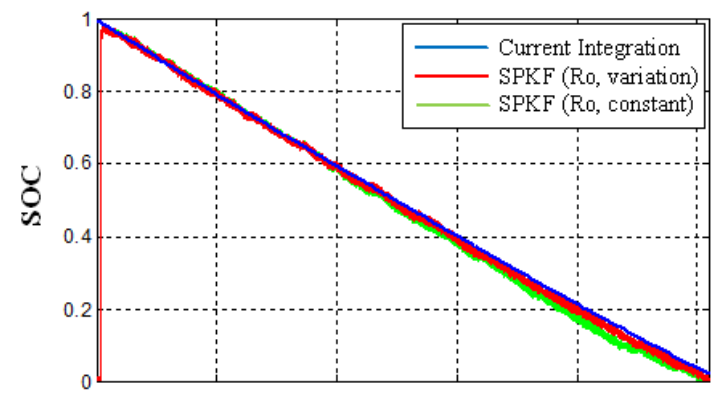

(b)

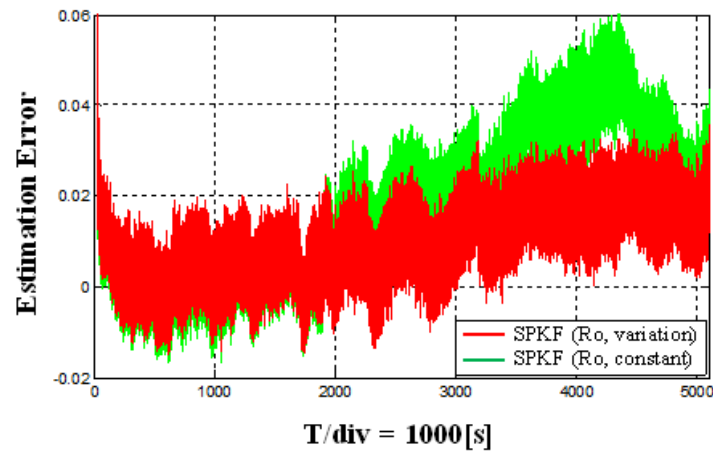

Fig. 12. Performance of estimated SOC (experiment). (a) SOC values. (b) Errors.

Fig. 11 shows the estimated $C_{\text {capacity }}$ in Farad. Note that the instantaneous estimation is not required for predicting the battery capacity. Thus the average value of the estimated $C_{\text {capacity }}$ during $500 \mathrm{~s}$ is adopted, which is much larger than the sampling time of $1 \mathrm{~s}$ for measuring the voltage and current in the case of the SOC estimation. The estimation accuracy refers to the integral calculation of the discharging current which is 3.14 Ah during the discharging process. By the proposed method, the maximum estimated capacity is $3.24 \mathrm{Ah}$ and the minimum is $3.09 \mathrm{Ah}$, where the estimation error is about $5 \%$.

\section{EXPERIMENTAL RESULTS}

The validity of the proposed method has also been demonstrated by experimental tests. The same test system as the one shown in Fig. 2 was used. The battery parameters used are shown in Table I. A TMS320VC33 digital signal processor was used for implementing the estimation algorithm. The discharging current and the terminal voltage were measured, and a sampling time of $1 \mathrm{~s}$ was used. (a)

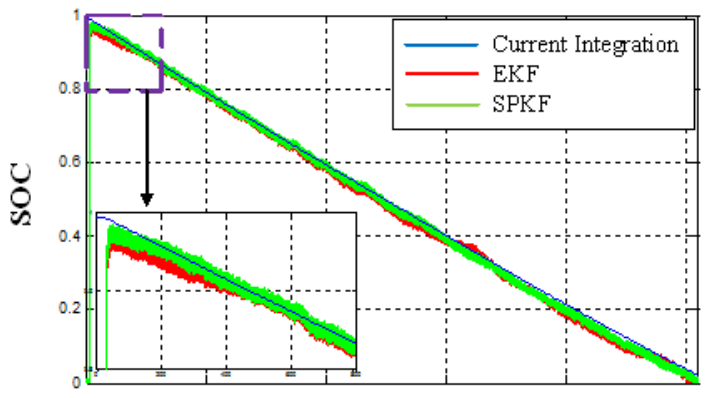

(b)

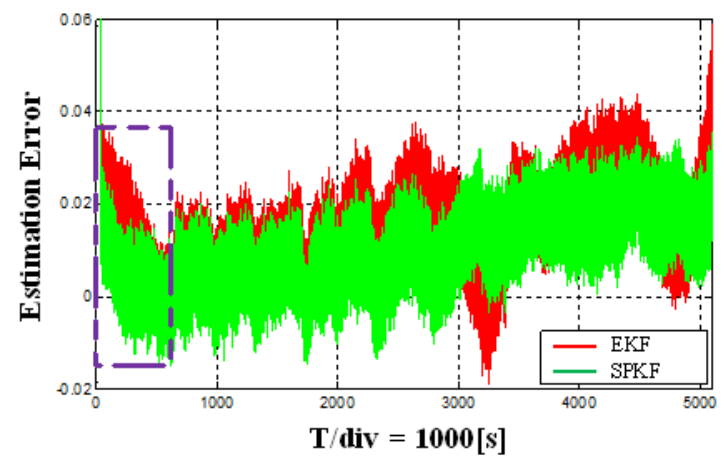

Fig. 13. Comparison of SOC estimation methods (experiment). (a) Estimated SOCs. (b) Estimation errors.

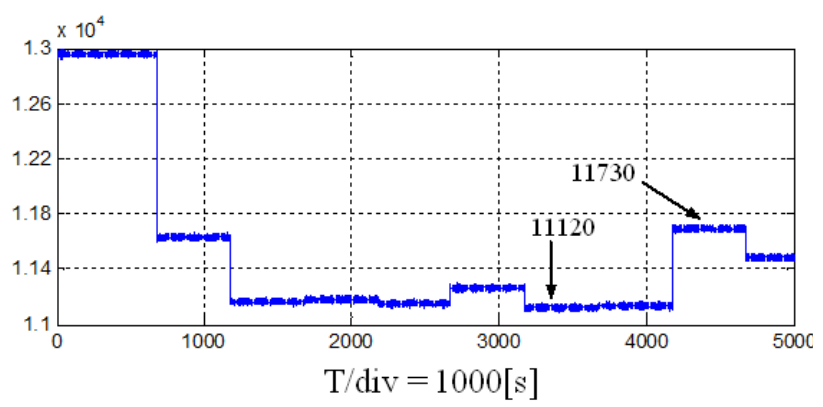

Fig. 14. Estimated $\mathrm{C}_{\text {capacity }}$

Fig. 12(a) and (b) show the results for estimating the SOC and its error, respectively, in which the two cases of using the constant and the variable $R_{o}$ are considered for the SPKF algorithm. The current integration method was also implemented to estimate the SOC for comparison. As can be seen in Fig. 12(b), the estimation error is increased after $2000 \mathrm{~s}$ due to the variation of $R_{0}$. In addition, the maximum error (3.5\%) in the case of a variable $R_{0}$ is lower than $6 \%$ in the case of a constant $R_{0}$.

The estimated SOCs and the estimation errors for the current integration method using the EKF and SPKF algorithms are shown in Fig. 13(a) and (b), respectively. The convergence time in the SPKF algorithm is about $350 \mathrm{~s}$ which is faster than the about $500 \mathrm{~s}$ in the EKF algorithm, as shown in Fig. 13(a). In addition, it can be seen from Fig. 13(b) that the performance of the SPKF algorithm is better than that of EKF, 
where the estimation error with the SPKF method is lower than that of the EKF method.

The estimated $C_{\text {capacity }}$ is shown in Fig. 14, which is the same as in the simulation case. The maximum and minimum estimated values are 3.26 $\mathrm{Ah}$ and $3.09 \mathrm{Ah}$, respectively, which is about a $5 \%$ error when compared with the actual value.

\section{CONCLUSIONS}

In this paper, a new estimation method for the SOC and the capacity of lithium polymer batteries using the SPKF has been proposed for the condition monitoring of batteries. With this algorithm, the measurement noise and disturbance can be compensated. In addition, the error of simple battery modeling is compensated by applying a variable series resistance $R_{o}$ for the SPKF. In ranges higher than $40 \%$ of the SOC, the estimation error is about $1.2 \%$ in the simulation and $1.5 \%$ in the experiment. For the capacity estimation, the estimation error is about $5 \%$ both for the simulation and experiment. Furthermore, the simulation and experimental results have shown that the convergence time of $300 \mathrm{~s}$ in the SPKF algorithm is faster than the $500 \mathrm{~s}$ in the EKF method.

\section{ACKNOWLEDGEMENT}

This work has been supported by KESRI (Korea Electrical Engineering and Science Research Institute) (2009T100100651), which is funded by MKE (Ministry of Knowledge Economy).

\section{REFERENCES}

[1] L. Maharjan, T. Yamagishi, H. Akagi, and J. Asakura, "Fault-tolerant operation of a battery-energy-storage system based on a multilevel cascade PWM converter with star configuration,” IEEE Trans. Power Electron., Vol. 25, No. 9, pp. 2386-2396, Sep. 2010.

[2] H. Qian, J. Zhang, J. S. Lai, and W. Yu, "A high-efficiency grid-tie battery energy storage system,” IEEE Trans. Power Electron., Vol. 26, No. 3, pp.886-896, Mar. 2011.

[3] I. Aharon and A. Kuperman, "Topological overview of powertrains for battery-powered vehicles with range extenders,” IEEE Trans. Power Electron., Vol. 26, No. 3 Mar. 2011.

[4] K.-H. Park, C.-H. Kim, H.-K. Cho, and J.-K. Seo, “Design considerations of a lithium ion battery management system (BMS) for the STSAT-3 satellite," Journal of Power Electronics, Vol. 10, No. 2, Mar. 2010.

[5] K. S. Ng, Y. F. Huang, C. S. Moo and Y. C. Hsieh, “An enhanced coulomb counting method for estimating state-ofcharge and state-of-health of lead-acid batteries," in Proc. IEEE INTELEC, pp. 1-5, Oct. 2009.

[6] S. Piller, M. Perrin, and A. Jossen, "Methods for state-ofcharge determination and their applications," Journal of Power Sources, Vol. 96, No. 1, pp. 113-120, Jun. 2001.
[7] N. A. Windarko and J. Choi, "SOC estimation based on OCV for NiMH batteries using an improved takacs model," Journal of Power Electronics, Vol. 10, No. 2, pp. 181-186, Mar. 2010.

[8] J.-H. Lee and W. Choi, "Novel state-of-charge estimation method for lithium polymer batteries using electrochemical impedance spectroscopy,” Journal of Power Electronics, Vol. 11, No. 2, pp. 237-243, Mar. 2011.

[9] B. S. Bhangu, P. Bentley, D. A. Stone, and C. M. Bingham, "Nonlinear observers for predicting state-of-charge and state-of-health of lead-acid batteries for hybrid electric vehicles," IEEE Trans. Veh. Technol., Vol. 54, No. 3, pp. 783-794, May 2005.

[10] M. Coleman, C. K. Lee, and W. G. Hurley, "State of health determination: two pulse load test for a VRLA battery," in Proc. IEEE PESC, pp. 1-6, Jun. 2006.

[11] D. Feder, T. Croda, K. Champlin, and M. Hlavac, "Field and laboratory studies to assess the state of health of valveregulated lead acid batteries. Conductance/capacity correlation studies," in Proc. IEEE INTELEC, pp. 218-233, Oct. 1992.

[12] M. Kniveton and A. I. Harrison, "Impedance/conductance measurements as an aid to determining replacement strategies," in Proc. IEEE INTELEC, pp. 297-301, Oct. 1988.

[13] I.-S. Kim, "A technique for estimating the state of health of lithium batteries through a dual-sliding-mode observer," IEEE Trans. Power Electron., Vol. 25, No. 4, pp. 10131022, Apr. 2010.

[14] C. R. Gould, C. M. Bingham, D. A. Stone, and P. Bentley, "New battery model and state-of-health determination through subspace parameter estimation and state-observer techniques," IEEE Trans. Veh. Technol., Vol. 58, No. 8, pp. 3905-3916, Oct. 2009.

[15] D. Haifeng, W. Xuezhe, and S. Zechang, “A new SOH prediction concept for the power lithium-ion battery used on HEVs," in Proc. IEEE VPPC, pp. 1649-1653, Sep. 2009.

[16] D. W. Dennis, V. S. Battaglia, and A. Belanger, "Electrochemical modeling of lithium polymer batteries," Journal of Power Source, Vol. 110, No. 2, pp. 310-320, Aug. 2002.

[17] D. Rakhmatov, S. Vrudhula, and D. A. Wallach, "A model for battery lifetime analysis for organizing applications on a pocket computer," IEEE Trans. Very Large Scale Integr. (VLSI) Syst., Vol. 11, No. 6, pp. 1019-1030, Dec. 2003.

[18] B. Schweighofer, K. M. Raab, and G. Brasseur, "Modeling of high power automotive batteries by the use of an automated test system," IEEE Trans. Instrum. Meas., Vol. 52, No. 4, pp. 1087-1091, Aug. 2003.

[19] L. Gao, S. Liu, and R. A. Dougal, "Dynamic lithium-ion battery model for system simulation," IEEE Trans. Compon. Packag. Technol., Vol. 25, No. 3, pp. 495-505, Sep. 2002.

[20] S. Buller, M. Thele, R. W. D. Doncker, and E. Karden, "Impedance-based simulation models of supercapacitors and li-ion batteries for power electronic applications," IEEE Trans. Ind. Applicat., Vol. 41, No. 3, May 2005.

[21] L. Benini, G. Castelli, A. Macii, E. Macii, M. Poncino, and R. Scarsi, "Discrete-time battery models for system-level low-power design,” IEEE Trans. Very Large Scale Integr. (VLSI) Syst., Vol. 9, No. 5, Oct. 2001.

[22] M. Chen, and G. A. Rincon-Mora, "Accurate electrical battery model capable of predicting runtime and I-V performance,” IEEE Trans. Energy Convers., Vol. 21, No. 2, pp. 504-511, Jun. 2006. 
[23] G. L. Plett, “Sigma-point Kalman filtering for battery management systems of LiPB-based HEV battery packs Part 2: Simultaneous state and parameter estimation," Journal of Power Sources, Vol. 161, No. 2, pp. 1369-1384, Oct. 2006.

[24] L. Wang, L. Wang, C. Liao, and J. Liu, "Sigma-point Kalman filter application on estimating battery SOC," in Proc. IEEE VPPC, pp. 1592-1595, Sep. 2009.

[25] E. Ghahremani and I. Kamwa, "Online state estimation of a synchronous generator using unscented Kalman filter from phasor measurement units," IEEE Trans. Energy Convers., Vol. 26, No. 4, pp. 1099-1108, Dec. 2011.

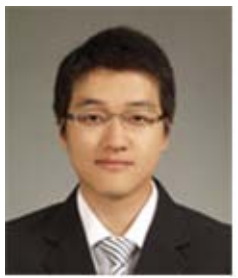

Bo-Hwan Seo was born in 1982. He received his B.S. and M.S. in Electrical Engineering from Yeungnam University, Gyeongsan, Korea, in 2008 and 2011, respectively. He is currently with LG Electronics Inc., Changwon, Korea. His current research interests include battery management systems, control of power converters, renewable energy systems, and motor drives.

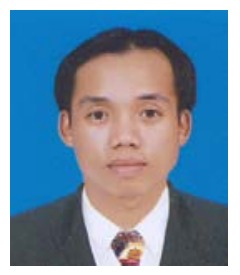

Thanh Hai Nguyen was born in Dong Thap, Vietnam. He received his B.S. in Engineering from the Technology University of Ho Chi Minh City, Vietnam, in 2003, and his M.S. in the Department of Electrical Engineering, Yeungnam University, Gyeongbuk, Korea, in 2010, where he is currently working toward his Ph.D. He was an Assistant Lecturer in the College of Technology, Can Tho University, Vietnam, in 2003. His current research interests include power converters, machine drives, and wind power generation.

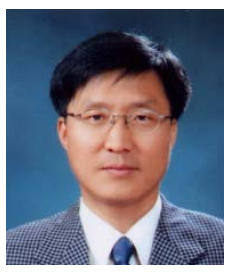

Dong-Choon Lee received his B.S., M.S., and Ph.D. in Electrical Engineering from Seoul National University, Seoul, Korea, in 1985, 1987, and 1993, respectively. He was a Research Engineer with Daewoo Heavy Industry from 1987 to 1988. Since 1994, he has been a Faculty Member in the Deptartment of Electrical Engineering, Yeungnam University, Gyeongbuk, Korea. He is currently serving as a Publication Editor for the Journal of Power Electronics, Korean Institute of Power Electronics, Seoul, Korea. As a Visiting Scholar, he joined the Power Quality Laboratory, Texas A\&M University, College Station, U.S.A., in 1998, the Electrical Drive Center, University of Nottingham, U.K., in 2001, the Wisconsin Electric Machines and Power Electronic Consortium, University of Wisconsin, Madison, U.S.A., in 2004, and the FREEDM Systems Center, North Carolina State University, Raleigh, U.S.A., in 2011-2012. His current research interests include ac machine drives, control of power converters, wind power generation, and power quality.

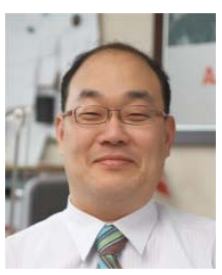

Kyo-Beum Lee was born in Seoul, Korea, in 1972. He received his B.S. and M.S. in Electrical and Electronic Engineering from Ajou University, Suwon, Korea, in 1997 and 1999, respectively. He received his Ph.D. in Electrical Engineering from Korea University, Seoul, Korea in 2003. From 2003 to 2006, he was with the Institute of Energy Technology, Aalborg University, Aalborg, Denmark. From 2006 to 2007, he was with the Department of Electronics and Information Engineering, Chonbuk National University, Jeonju, Korea. In 2007 he joined the School of Electrical and Computer Engineering, Ajou University, Suwon, Korea. He is an Associated Editor of the IEEE Transactions on Power Electronics, the IEEE Transactions on Industrial Electronics and the Journal of Power Electronics. His current research interests include electric machine drives and renewable power generation.

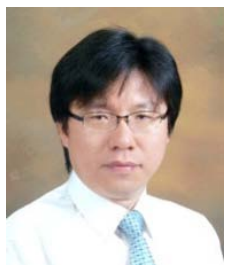

Jang-Mok Kim received his B.S. from Pusan National University, Busan, Korea, in 1988, and his M.S. and Ph.D. from the Department of Electrical Engineering, Seoul National University, Seoul, Korea, in 1991 and 1996, respectively. From 1997 to 2000, he was a Senior Research Engineer with the Korea Electrical Power Research Institute (KEPRI). Since 2001, he has been with the School of Electrical Engineering, Pusan National University (PNU), where he is currently a Faculty Member. In addition, he is a Research Member of the Research Institute of Computer Information and Communication, and the director of PNU-LG smart control center at PNU. His current research interests include the control of electric machines, electric vehicle propulsion, and power quality. 